\title{
A Review of the Long-Term Oncologic Outcomes of Robotic Surgery Versus Laparoscopic Surgery for Colorectal Cancer
}

\author{
Fatima G. Wilder ${ }^{1}$ - Atuahni Burnett ${ }^{1} \cdot$ Joseph Oliver $^{1} \cdot$ Michael F. Demyen ${ }^{2}$. \\ Ravi J. Chokshi ${ }^{3}$
}

Received: 12 May 2015 / Accepted: 12 October 2015 /Published online: 22 October 2015

(C) Association of Surgeons of India 2015

\begin{abstract}
The goal of this review was to compare long-term oncologic outcomes of robotic versus laparoscopic resection of colorectal cancer. A literature search was performed using PubMed, EMBASE, Cochrane, and Medline (2002-2014). Search terms: laparoscopic, robotic, rectal, colon, surgery, oncologic, and outcomes. Studies comparing overall and disease free survival of robotic versus laparoscopic surgery for colorectal cancer were included. Meta-analysis was performed using OpenMeta[Analyst] for Windows 8. Five studies were identified reporting on overall survival, disease free survival, lymph node extraction, and distal and circumferential resection margin. Three hundred and seventeen patients underwent robotic resection and 368 underwent laparoscopic resection, with similar demographics. Operative times were longer with robotic resections, with no difference in estimated blood loss (EBL) or length of stay. The disease stage was distributed similarly in both groups. Similar numbers underwent neoadjuvant therapy. Laparoscopic resection was associated with $3.2 \mathrm{~mm}$ larger distal resection margins $(p=0.04)$ and 2.2 more lymph nodes removed $(p=0.001)$, but with equivalent circum-
\end{abstract}

**Abstract presented as a QuickShot presentation at the Academic Surgical Congress 2015 Annual Meeting in Las Vegas, Nevada (Feb 2-5).

Fatima G. Wilder

fatima.garuba9@gmail.com

1 Department of Surgery, Rutgers-New Jersey Medical School, 185 South Orange Avenue, Newark, NJ 07101, USA

2 Department of Medicine, Rutgers-New Jersey Medical School, 90 Bergen St, DOC 2100, Newark, NJ 07103, USA

3 Rutgers-New Jersey Medical School, University Hospital Cancer Center, 205 South Orange Avenue, G-1228, Newark, NJ 07103, USA ferential resection margin status. Disease-free and overall survival was equivalent. Robotic and laparoscopic surgery for colorectal cancer offer comparable overall and disease free survival. Laparoscopic surgery offered a slight advantage in operative time, distal margin, and lymph node yield. Larger, prospective trials are needed to confirm the equivalence of these approaches.

Keywords Robotic colorectal surgery $\cdot$ Oncologic outcomes

\section{Introduction}

The robot was first introduced to the surgical arena in the 1980s in the form of telesurgery [1]. Through several modifications and advancements since then, robotic surgery has established itself as a valid option for patients undergoing certain surgical procedures. This first became widely adopted in the fields of urologic and gynecologic surgery, however, appropriate usage within these fields remains a hotly debated topic [2-4]. Since the report of the first robotic right hemicolectomy and sigmoid colectomy in 2002, the robot has established itself as an alternative approach to laparoscopy in colorectal surgery that is worth considering $[2,5]$. Despite this, there remains an understandable degree of skepticism regarding the robot's ability and feasibility to replace laparoscopic surgery for certain procedures.

A large breadth of literature has been published comparing the benefits of robotic and laparoscopic surgery for colorectal disease and the data thus far suggests that robotic colorectal surgery (RCS) is safe and feasible. Laparoscopic colorectal surgery (LCS) offers an advantage over RCS when one looks at operative time, the steep learning curve, and operative costs $[5,6]$. However, the shortcomings of laparoscopic surgery for colorectal procedures have also been highlighted, with the 
most commonly cited examples being the limited range of motion with only four degrees of freedom, loss of dexterity, and two-dimensional visualization [2, 7-10]. The robot addresses some of these shortcomings by offering seven degrees of freedom, three-dimensional imaging, tremor filtration, more precise pelvic dissection, greater surgeon comfort, and a stable control camera [2, 7-9]. Data further suggests that RCS offers decreased post-operative pain, lower intraoperative conversion rates, shorter time to resume diet, and shorter length of hospital stay [6, 9]. Additionally, RCS may offer an advantage in dissection in the deep pelvis especially in patients who have undergone pre-operative chemotherapy $[2,9]$. While each surgical method offers some advantages over the other, several studies have demonstrated comparable outcomes between robotic and laparoscopic colorectal surgery for intra-operative morbidity, complication rates, and postoperative recovery $[1,9,11,12]$.

While there is sufficient early data to make preliminary comparisons of outcomes of RCS versus LCS, the data is limited in regard to long-term oncologic outcomes. Outcomes such as lymph node (LN) extraction, circumferential resection margin (CRM), and distal resection margin (DRM) have been reviewed by some and shown to be comparable between RCS and LCS. However, little has been published comparing overall survival (OS) and disease-free survival (DFS) for the two methods. We sought to review the literature published thus far looking specifically at OS and DFS in RCS versus LCS in patients with colon and rectal cancer.

\section{Materials and Methods}

An electronic search was performed using Medline, PubMed, EMBASE and Cochrane. Keywords used were: laparoscopic, robotic, rectal, colon, surgery, oncologic, and outcomes. Selection of studies was limited to those published between 2002 and 2014. The PubMed search identified 18 studies, EMBASE identified 38 studies, Medline identified 16 studies, and no studies were identified through Cochrane giving us a total of 72 potential studies for review. We further expanded our search by reviewing the bibliographies of related studies for potentially relevant papers; however, this did not lead to the identification of any additional studies. There were a total of seven studies that reviewed long-term oncologic outcomes for resection of colon and rectal cancer, however two of those did not directly compare outcomes of laparoscopic and robotic resection within the same study conditions, thus they were excluded, leaving us with five studies for inclusion. The studies were reviewed by a single reviewer.

All studies published on robotic surgery, with a comparison to laparoscopic surgery, were reviewed in full. Inclusion criteria were: direct comparison of long-term oncologic outcomes with laparoscopic versus robotic resection of a malignancy of the colon or rectum, totally robotic resection or a hybrid operation (laparoscopic mobilization of the left colon and the splenic flexure), and publication in English. Exclusion criteria were: failure to report overall survival and disease free survival, and resection for benign diseases of the colon and rectum.

Data extracted from each study, when available, was: type of study, number of subjects ( $n$ ), demographics (age, gender), and TNM staging. Further, the following data was collected: LN yield, length of stay (LOS), BMI, estimated blood loss (EBL), length of surgery (time), CRM, DRM, OS, and DFS.

Hybrid operations were evaluated as robotic resections because the portion of surgery that is directly involved in oncologic resection, specifically the total mesorectal excision (TME) in rectal surgery, is performed by the robot. The preceding portions, namely, mobilization of the splenic flexure and left colon, are performed laparoscopically.

\section{Statistical Analysis}

Using a standard spreadsheet, the data from all studies were combined by calculating a weighted average. The statistics regarding lymph nodes extracted, and resection margins were compared using a random-effects model based on the maximum likelihood method. Heterogeneity was assessed with standard Chi-square and expressed as $\mathrm{I}^{2}$ values. Metaanalysis was performed using OpenMeta[Analyst] for Windows 8 (based on $\mathrm{R}$ version 3.0.2 and the metaphor package) $[13,14]$. The standard deviations for the survival data were calculated based on a standard error of proportion calculation. Synthesis of the 3-year OS and DFS were also calculated by a weighted average method, however, the Park study was excluded due to its 5-year endpoint.

\section{Results}

Seventy-two articles were identified, 67 of which were excluded. The final analysis included five studies with a total $n$ of 685 patients; of these, 317 patients underwent hybrid robotic resection and 368 underwent laparoscopic resection [8, 15-18]. Patient demographics and perioperative variables are presented in Table 1. There were no differences between groups with regard to gender and BMI, however, the RCS group was slightly younger in age (60.0 vs. $61.8, p=0.04)$. Rates of neo-adjuvant chemoradiotherapy were also similar between groups ( $\mathrm{RCS} 41.0 \%$ vs. $39.6 \%, p=0.83$ ). Operative times were significantly longer with RCS compared to LCS (269.4 min vs. $245.4 \mathrm{~min}, p<0.001$ ), however, EBL (121.9 vs $129.5, p=0.40)$ and length of hospital stay (7.1 vs. $7.3, p=0.43$ ) were not statistically different between groups.

The most common surgeries performed were low anterior resection (LAR). In the Park and Lim studies, all patients 
Table 1 Patient demographic and perioperative outcomes

\begin{tabular}{llll}
\hline & RCS & LCS & $P$ value \\
\hline \% Male & $63.4 \%$ & $63.3 \%$ & 0.98 \\
Age (years) & $60.0( \pm 11.4)$ & $61.8( \pm 11.3)$ & 0.04 \\
BMI & $23.5( \pm 3.2)$ & $23.5( \pm 3.6)$ & $>0.99$ \\
Pre-op CRT (\%) & $41.0( \pm 3.2)$ & $39.6( \pm 3.2)$ & 0.83 \\
OR Time (min) & $269.4( \pm 84.1)$ & $245.4( \pm 70.0)$ & $<0.001$ \\
EBL (ml) & $121.9( \pm 130.2)$ & $129.5( \pm 101.2)$ & 0.40 \\
LOS (days) & $7.1( \pm 3.0)$ & $7.3( \pm 3.7)$ & 0.43 \\
\hline
\end{tabular}

Standard deviations listed in brackets. $P$ values in italics are significant

underwent LAR. In the study by Saklani, $71.9 \%$ of patients in the LCS group underwent LAR as compared to $62.2 \%$ in the RCS. Patriti reported use of a partial mesorectal excision (PME) where the rectum and mesorectum was excised $4 \mathrm{~cm}$ below the lower end of the tumor after sharp perimesorectal dissection. A total of $64.8 \%$ of their patients in the LCS group underwent PME as compared to $10.3 \%$ in the RCS. Conversely, $62.8 \%$ of the patients in the RCS group underwent TME as compared to $21.6 \%$ who underwent PME. Baek utilized a combined ultra-low anterior resection and coloanal anastomosis in all patients. Two studies reported use of inter-sphincteric resections (ranging from 1-10\% in the LCS group and $4-12.1 \%$ in the RCS group) and coloanal anastomoses (5-22 \% in the LCS group and $17.2-31.1 \%$ in the RCS group).

\section{Oncologic Outcomes}

All studies were comparable in the distribution of patients by stage. Only the Baek 2013 study reported patients that were considered Stage 0 with $10.8 \%$ in the LCS group and $12.8 \%$ in the RCS group. No clear explanation was given as to how this stage was classified.

Using our random-effects model, RCS groups were compared with corresponding LCS groups in the same study. LCS were found to produce 2.17 more lymph nodes, which was significant with a $p=0.001$ (Fig. 1). The same analysis was used to examine distance to distal resection margin in Fig. 2. The LCS group was found to have a $3.2 \mathrm{~mm}$ longer distance to the distal resection margin, which was statistically significant with a $p=0.04$ (Fig. 2). Finally, the risk of having a positive circumferential resection margin was analyzed using the random-effects model as above and the odds ratio of RCS:LCS was 1.08 with $p=0.86$, demonstrating no significant difference, Fig. 3. None of these analyses had any significant heterogeneity.

The 3-year disease-free survival (DFS) and overall survival (OS) for each study is listed in Table 2 along with the mean DFS and OS generated using a weighted average approach. Mean 3-year DFS for RCS was $84.1 \%( \pm 4.4 \%)$ and for LCS was $86.9 \%( \pm 3.8 \%)$ with no significant difference $(p=0.93)$. Mean 3-year OS for RCS was $90.5 \%( \pm 4.1)$ and for LCS was $93.3 \%( \pm 2.8 \%)$ with no significant difference $(p=0.34)$. The Park study reported survival at 5 years, and thus was excluded from the mean calculation.

\section{Discussion}

The potential benefits of robotic surgery in colon and rectal cancer resection are of increasing interest to colorectal surgeons. Our review demonstrates that in comparison to laparoscopic surgery, robotic surgery was associated with slightly smaller resection margins, resection of fewer nodes, and equivalent odds of a positive circumferential resection margin. More importantly, a review of the data suggests that a 3-5 year overall and disease-free survival are comparable between robotic and laparoscopic resection of sigmoid and rectal cancer.

Many studies have been published comparing outcomes of robotic versus laparoscopic resection of colorectal cancers. Published data suggests that the robot offers comparable outcomes in estimated blood loss (EBL), hospital LOS, and conversions and complications rate. Two areas that initially

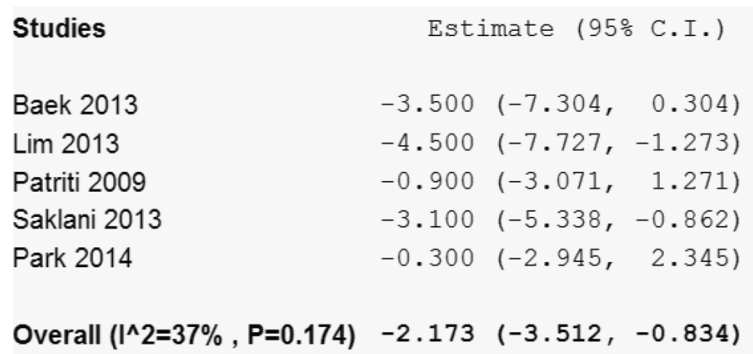

Fig. 1 Lymph node yield is better with laparoscopic compared to robotic resection. The difference in lymph node yields between robotic and lap (RCS-LCS) is listed for each study and for the combined overall. The

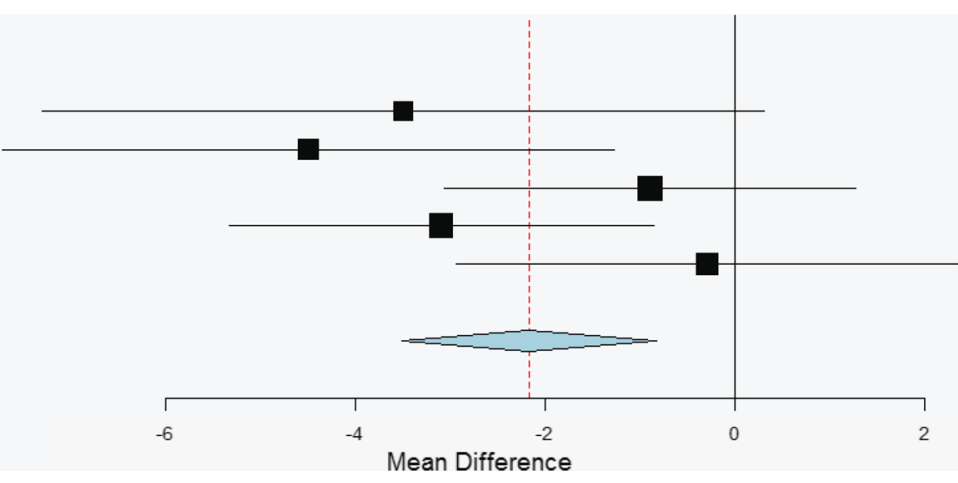

LCS group produced on average 2.17 more lymph nodes, which was a statistically significant improvement in yield compared to RCS $(p=0.001)$. No significant heterogeneity 


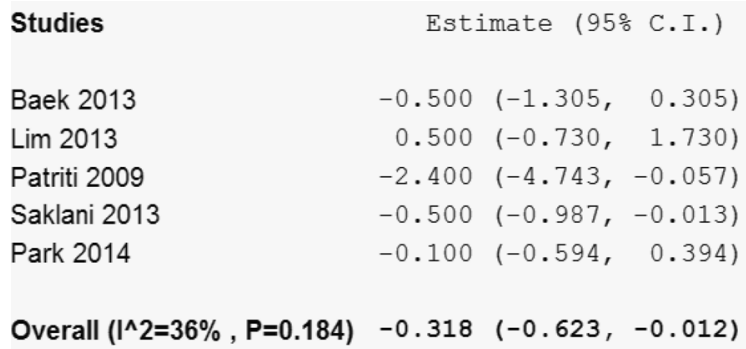

Fig. 2 Laparoscopic procedures achieve a larger distal resection margin. The difference in distance of tumor to the resection margin between robotic and lap (RCS-LCS) procedures are listed for each study and for

showed worse outcomes for robotic surgery were length of surgery (time) and cost. With regard to length of surgery, the early differences seen are beginning to equalize as surgeons become more comfortable with the robot and the learning curve decreases [7, 9]. Yang et al. performed a meta-analysis comparing robot-assisted versus conventional laparoscopic surgery for rectal cancer in 2012. They found that when looking at surgery performed for malignant colorectal diseases, there was an average of 21.6 min spent longer with robotic resection. However, other meta-analyses found that any difference in time between the two groups was not statistically significant $[9,19]$. Despite the benefits offered by the robot, the persistently high costs remain a significant difference between it and laparoscopic surgery. It remains yet to be demonstrated that the benefits offered by the robot outweigh the additional costs incurred by its use $[9,16,19]$.

\section{Oncologic Outcomes}

One operative factor that is known to have a significant effect on oncologic outcomes is lymph node extraction. Complete mesocolic excision along with lymphadenopathy has been shown to be associated with better oncologic outcomes [7]. The recommendation by the College of American Pathologists was a minimum of 12 lymph nodes for colorectal resection. This has been accepted by the American Joint Commission on Cancer and the National Cancer Institute and has been used as a standard to guide therapy [19-21]. Three meta-analyses compared the difference in the number of nodes extracted robotically versus laparoscopically. One of these, by Yang et al., looked at lymph node excision for colorectal disease. The other two by Kim et al. and Trastulli et al. looked specifically at resection for rectal cancer [22, 23]. None of the three studies found a statistically significant difference in the number of nodes extracted by the robot as compared to laparoscopic resection. However, in our analysis, we found that the robot resected an average of 2.17 fewer nodes, a value that was statistically significant $(p=0.001)$. Our findings may be inconsistent with prior studies in this regard due to the small number of patients analyzed. If the weighted averages were examined, however, RCS resected a mean of $13.4( \pm 7.5)$ nodes compared to $15.4( \pm 9.5)$ lymph nodes with LCS resection. As this value is above the current standard recommendations, it may not have an effect on overall survival outcomes as noted by the survival data in our analysis.

Another operative factor that has been found to have significant effects on patient outcomes is the quality of resection. Total mesorectal excision (TME) is the gold standard for rectal surgery. It has been found that a complete TME is associated with decreased local recurrence rates. Additionally, a negative

\begin{tabular}{|c|c|c|c|c|}
\hline Studies & Estimate 195 & 5\% C.I.) & Ev/Trt & $\mathrm{Ev} / \mathrm{Ctrl}$ \\
\hline Baek 2013 & $4.059(0.404$, & $40.734)$ & $3 / 37$ & $1 / 47$ \\
\hline Patriti 2009 & $0.787 \quad 0.015$ & $40.835)$ & $0 / 37$ & $0 / 29$ \\
\hline Saklani 2013 & $0.360 \quad(0.036$ & $3.549)$ & $1 / 64$ & $3 / 71$ \\
\hline Park 2014 & $1.060(0.363$, & $3.093)$ & $6 / 84$ & $9 / 1$ \\
\hline Overall $\left(I^{\wedge} 2=0 \%, P=0.536\right)$ & $1.082(0.452$, & $2.588)$ & $10 / 222$ & $13 / 2$ \\
\hline
\end{tabular}

Fig. 3 Odds ratio of robotic procedures having a positive CRM compared to laparoscopic procedures (RCS/LCS) are listed for each study and for the combined overall. No difference in risk of positive

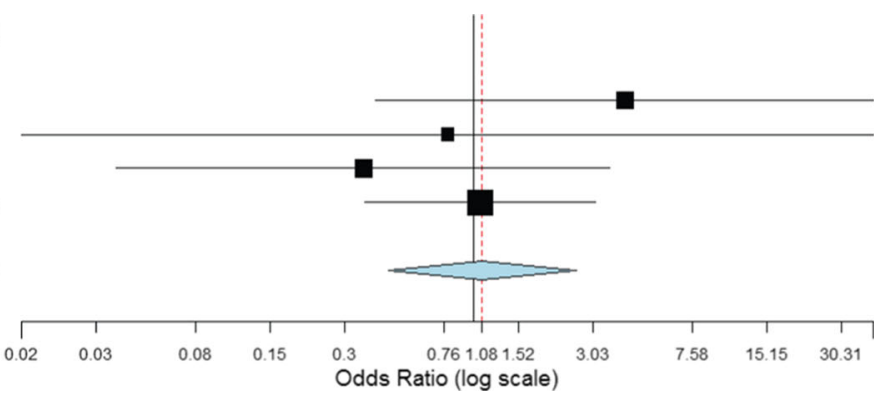

circumferential resection margin (CRM) between the two groups was found $(p=0.86)$. No significant heterogeneity 
Table 2 Disease free and overall survival

\begin{tabular}{llllll}
\hline Author, year & DFS & & & OS \\
\cline { 2 - 3 } \cline { 5 - 6 } & LCS & RCS & & LCS & RCS \\
\hline Patriti & $94.6( \pm 3.7)$ & $100( \pm 0.0)$ & & $97.2( \pm 2.7)$ & $96.5( \pm 3.4)$ \\
Baek & $81.2( \pm 6.4)$ & $80.6( \pm 5.8)$ & & $90.7( \pm 4.8)$ & $86.5( \pm 4.9)$ \\
Lim & $90.0( \pm 2.5)$ & $89.2( \pm 5.3)$ & & $93.5( \pm 2.0)$ & $92.1( \pm 4.6)$ \\
Saklani & $78.8( \pm 5.1)$ & $77.7( \pm 4.8)$ & & $92.1( \pm 3.4)$ & $90.0( \pm 3.5)$ \\
Park* & $78.7( \pm 4.5)$ & $81.9( \pm 3.3)$ & & $93.5( \pm 2.7)$ & $92.8( \pm 2.2)$ \\
Mean & $86.9( \pm 3.8)$ & $84.1( \pm 4.4)$ & & $93.3( \pm 2.8)$ & $90.5( \pm 4.1)$ \\
$p$ values & 0.93 & & & 0.34 &
\end{tabular}

Survival listed is 3-year data except with Park study, which is 5-year survival. Standard deviations listed in brackets. Mean values listed in italics

*Excluded from mean due to 5-year end point

CRM (defined as $>1 \mathrm{~mm}$ resection margin) is associated with decreased rates of distant metastases and improved survival $[20,24]$. A CRM of $>2 \mathrm{~mm}$ is associated with lower local recurrence rates [25]. Current literature shows that when looking at the percentage of positive CRM there is a range of 0-7.5 \% with LCS and 5-7.3\% with RCS. One metaanalysis by Xiong et al. looking at RCS versus LCS performing TME for rectal cancer, found that robotic TME was associated with lower rates of positive CRM as compared to laparoscopic TME $(p=0.04)$. The difference in DRM was not found to be statistically significant in that study $(p=0.36)$ [26]. The meta-analyses performed by Yang et al. and Trastulli et al. found no statistically significant difference in positive CRM or DRM between the two groups. We similarly found no significant difference in percentage of positive CRM, but there was a significantly larger DRM with the LCS group. Recent studies recommend a DRM of at least $2 \mathrm{~mm}$, a distance that was achieved in both the RCS and LCS groups if the weighted averages are examined $(2.5 \mathrm{~cm} \pm 1.8 \mathrm{~cm}$ and $3.6 \mathrm{~cm} \pm 2.7 \mathrm{~cm}$, respectively) [25]. This, therefore, suggests that while LCS may achieve larger margins in our analysis, both LCS and RCS meet the baseline recommendations.

The DFS and OS associated with RCS and LCS have been reported separately in recent literature. However, our review has been able to analyze studies that were a direct comparison between the two groups within one study set. In the four studies looking at outcomes at 3 years, we found a mean DFS of $86.9 \%$ with LCS and $84.1 \%$ with RCS. Mean OS with LCS was $93.3 \%$ as compared to $90.5 \%$ with RCS. The study by Park et al. was the only study identified that looked at outcomes at 5 years. They found a DFS of $78.7 \%$ with laparoscopic surgery and $81.9 \%$ with robotic surgery-both of which are slightly lower than our calculated mean at 3 years. OS with LCS was $93.5 \%$ and $92.8 \%$ with RCS for the Park study. Without the individual patient level data, we were unable to combine survival data from these studies to include it into our meta-analysis. However, our findings are consistent with prior reports and suggest that at 3 years, DFS and OS are similar in patients undergoing RCS and LCS. Additional studies are needed to determine the exact fate of patients at 5 years.

In the systematic review by Kim et al., they identified two studies looking at DFS and OS when only robotic resection was performed. These studies (excluded from our analysis due to their limited analysis of RCS alone) found a 3 year DFS of $73.7 \%$ (at a mean follow up of 20.2 months) and $77.6 \%$ (mean follow up 17.4 months). OS in these two studies were 96.2 and $97 \%$, respectively. However, given the lack of inclusion of LCS data in their study, it is difficult to directly compare Kim et al. with studies that include LCS data.

There is currently an international, randomized, multi-center, prospective trial comparing the two treatment modalities for rectal cancer. The ROLARR trial began enrollment in 2011 and is set to include 20 centers from approximately 8 countries. The primary outcome measure will be the rate of conversion to open operation. They will additionally compare the CRM and pathological involvement rates, the short- and longterm local recurrence rates, as well as long-term DFS and OS. The results of this study are greatly anticipated and will provide a significant amount of potentially useful and definitive information about the benefits of robotic surgery for rectal cancer as compared to laparoscopic surgery [27].

\section{Limitations}

The primary limitation of our review is the small study population available for analysis. As more studies are performed with longer-term oncologic outcomes reported, one will be better able to assess the effect of RCS on DFS and OS and evaluate these differences separately in rectal and colon cancer. Another limitation is the quality of studies that were included in the analysis. None of the studies were randomized control trials, thus suggesting that studies were affected by bias. For example, Patriti's study reports deliberately treating a larger number of low rectal cancers with a robotic approach because after the first few cases they felt a subjective improvement in low mesorectal dissection so randomization was abandoned. The Saklani data was reviewed retrospectively from a prospectively collected database, thus allowing for an inherent bias in patient selection.

\section{Conclusions}

This review suggests that RCS offers comparable oncologic outcomes to LCS at 3-year follow-up. However, it is important to consider factors affecting a potential bias including appropriate patient inclusion/exclusion and the use and timing 
of chemoradiation therapy. Data is still too limited to quantify significance in the differences between the two groups with regard to DFS and OS. There is a need for randomization of patients in a blinded study directly comparing robotic and laparoscopic resection for colon and rectal cancers. We additionally need to wait for a longer-term follow up to be available from the larger studies that have been performed.

\section{Compliance with Ethical Standards}

Conflict of Interest The authors declare that they have no competing interests.

\section{References}

1. Hussain A, Malik A, Halim MU, Ali AM (2014) The use of robotics in surgery: a review. Int J Clin Pract 68:1376-1382

2. Leong QM, Kim SH (2011) Robot-assisted rectal surgery for malignancy: a review of current literature. Ann Acad Med Singap 40: 460-466

3. Advincula AP, Song A (2007) The role of robotic surgery in gynecology. Curr Opin Obstet Gynecol 19:331-336

4. Guru KA, Hussain A, Chandrasekhar R, Piacente P, Hussain A, Chandrasekhar R, Piacente P, Bienko M, Glasgow M, Underwood W, Wilding G, Mohler JL, Menon M, Peabody JO (2009) Current status of robot-assisted surgery in urology: a multi-national survey of 297 urologic surgeons. Can J Urol 16: 4736-4741, discussion 4741

5. Kang J, Yoon KJ, Min BS, Hur H, Baik SH, Kim NK, Lee KY (2013) The impact of robotic surgery for mid and low rectal cancer: a case-matched analysis of a 3-arm comparison-open, laparoscopic, and robotic surgery. Ann Surg 257:95-101

6. Tam MS, Abbass M, Abbas MA (2014) Robotic-laparoscopic rectal cancer excision versus traditional laparoscopy. JSLS 18. doi:10. 4293/JSLS.2014.00020

7. Yang Y, Wang F, Zhang P, Shi C, Zou Y, Qin H, Ma Y (2012) Robot-assisted versus conventional laparoscopic surgery for colorectal disease, focusing on rectal cancer: a meta-analysis. Ann Surg Oncol 19:3727-3736

8. Saklani AP, Lim DR, Hur H, Min BS, Baik SH, Lee KY, Kim NK (2013) Robotic versus laparoscopic surgery for mid-low rectal cancer after neoadjuvant chemoradiation therapy: comparison of oncologic outcomes. Int J Color Dis 28:1689-1698

9. Fung AK, Aly EH (2013) Robotic colonic surgery: is it advisable to commence a new learning curve? Dis Colon Rectum 56:786-796

10. Baek SK, Carmichael JC, Pigazzi A (2013) Robotic surgery: colon and rectum. Cancer J 19:140-146

11. Kwak JM, Kim SH, Kim J, Son DN, Baek SJ, Cho JS (2011) Robotic vs laparoscopic resection of rectal cancer: short-term outcomes of a case-control study. Dis Colon Rectum 54:151-156

12. Yoo BE, Cho JS, Shin JW, Lee DW, Kwak JM, Kim J, Kim SH (2014) Robotic versus laparoscopic intersphincteric resection for low rectal cancer: comparison of the operative, oncological, and functional outcomes. Ann Surg Oncol 22(4):1219-1225

13. Viechtbauer W (2010) Conducting meta-analyses in R with the metafor package. J Stat Softw 36:1-48
14. Wallace BC, Dahabreh IJ, Trikalinos TA, Lau J, Trow P, Schmid $\mathrm{CH}$ (2012) Closing the gap between methodologists and end-users: $\mathrm{R}$ as a computational back-end. J Stat Softw 49:1-15

15. Patriti A, Ceccarelli G, Bartoli A, Spaziani A, Biancafarina A, Casciola L (2009) Short- and medium-term outcome of robotassisted and traditional laparoscopic rectal resection. JSLS 13: 176-183

16. Baek SJ, Al-Asari S, Jeong DH, Hur H, Min BS, Baik SH, Kim NK (2013) Robotic versus laparoscopic coloanal anastomosis with or without intersphincteric resection for rectal cancer. Surg Endosc 27: 4157-4163

17. Lim DR, Min BS, Kim MS, Alasari S, Kim G, Hur H, Baik SH, Lee KY, Kim NK (2013) Robotic versus laparoscopic anterior resection of sigmoid colon cancer: comparative study of long-term oncologic outcomes. Surg Endosc 27:1379-1385

18. Park EJ, Cho MS, Baek SJ, Hur H, Min BS, Baik SH, Lee KY, Kim NK (2014) Long-term oncologic outcomes of robotic low anterior resection for rectal cancer: a comparative study with laparoscopic surgery. Ann Surg 261(1):129-137

19. Compton CC, Fielding LP, Burgart LJ, Conley B, Cooper HS, Hamilton SR, Hammond ME, Henson DE, Hutter RV, Nagle RB, Nielsen ML, Sargent DJ, Taylor CR, Welton M, Willett C (2000) Prognostic factors in colorectal cancer. College of American Pathologists Consensus Statement 1999. Arch Pathol Lab Med 124:979-994

20. Wasserberg N, Gutman H (2008) Resection margins in modern rectal cancer surgery. J Surg Oncol 98:611-615

21. National Cancer Institute. Rectal cancer treatment: treatment option overview. 2014 [cited 2014 11/11/2014]; Available from: http:// www.cancer.gov/cancertopics/pdq/treatment/rectal/ HealthProfessional/page4

22. Kim NK, Baik SH, Seong JS, Kim H, Roh JK, Lee KY, Sohn SK, Cho CH (2006) Oncologic outcomes after neoadjuvant chemoradiation followed by curative resection with tumor-specific mesorectal excision for fixed locally advanced rectal cancer: impact of postirradiated pathologic downstaging on local recurrence and survival. Ann Surg 244:1024-1030

23. Trastulli S, Farinella E, Cirocchi R, Cavaliere D, Avenia N, Sciannameo F, Gulla N, Noya G, Boselli C (2012) Robotic resection compared with laparoscopic rectal resection for cancer: systematic review and meta-analysis of short-term outcome. Color Dis 14:e134-e156

24. Nagtegaal ID, van Krieken JH (2002) The role of pathologists in the quality control of diagnosis and treatment of rectal cancer-an overview. Eur J Cancer 38:964-972

25. Nagtegaal ID, Marijnen CA, Kranenbarg EK, van de Velde CJ, van Krieken JH, Pathology Review C, Cooperative Clinical I (2002) Circumferential margin involvement is still an important predictor of local recurrence in rectal carcinoma: not one millimeter but two millimeters is the limit. Am J Surg Pathol 26:350-357

26. Xiong B, Ma L, Zhang C, Cheng Y (2014) Robotic versus laparoscopic total mesorectal excision for rectal cancer: a meta-analysis. J Surg Res 188:404-414

27. Collinson FJ, Jayne DG, Pigazzi A, Tsang C, Barrie JM, Edlin R, Garbett C, Guillou P, Holloway I, Howard H, Marshall H, McCabe C, Pavitt S, Quirke P, Rivers CS, Brown JM (2012) An international, multicentre, prospective, randomised, controlled, unblinded, parallel-group trial of robotic-assisted versus standard laparoscopic surgery for the curative treatment of rectal cancer. Int J Color Dis 27:233-241 